\title{
Adaptive Disturbance Rejection Control for Automatic Carrier Landing System
}

\author{
Xin Wang, Xin Chen, and Liyan Wen \\ College of Automation Engineering, Nanjing University of Aeronautics and Astronautics, Nanjing 211100, China \\ Correspondence should be addressed to Xin Chen; chenxin@nuaa.edu.cn
}

Received 27 March 2016; Revised 25 June 2016; Accepted 5 July 2016

Academic Editor: Jean J. Loiseau

Copyright (C) 2016 Xin Wang et al. This is an open access article distributed under the Creative Commons Attribution License, which permits unrestricted use, distribution, and reproduction in any medium, provided the original work is properly cited.

\begin{abstract}
An adaptive disturbance rejection algorithm is proposed for carrier landing system in the final-approach. The carrier-based aircraft dynamics and the linearized longitudinal model under turbulence conditions in the final-approach are analyzed. A stable adaptive control scheme is developed based on LDU decomposition of the high-frequency gain matrix, which ensures closed-loop stability and asymptotic output tracking. Finally, simulation studies of a linearized longitudinal-directional dynamics model are conducted to demonstrate the performance of the adaptive scheme.
\end{abstract}

\section{Introduction}

The automatic carrier landing system requires that the aircraft arrives at the touchdown point in a proper sink speed and a small margin error for position. The key requirements of this problem are that the aircraft must remain within tight bounds on a three-dimensional flight path while approaching the ship and then touch down in a relatively small area with acceptable sink rate, angular attitudes, and speed. Further, this must be accomplished with limited control authority for varying conditions of wind turbulence and ship air wake.

During the past decades, research on the improvement of the automatic carrier landing system had received much attention. A vertical rate and vertical acceleration reference were used in the control law to reduce the turbulence effects and deck motion in [1]. A noise rejection filter was added to the control algorithm to decrease the sensitivity to noise and an optimization of the control gains was then performed to prevent degradation of the system's response to turbulence in carrier landing in [2]. A finite horizon technique was introduced to maintain a constant flight-path angle under the worst case conditions during carrier landing in [3]. An improvement in carrier landing performance was made by the incorporation of the direct lift control using $H_{\infty}$ outputfeedback synthesis [4]. As pointed out in [5], a fuzzy logic based carrier landing system was designed and the results indicated that fuzzy logic could yield significant benefits for aircraft outer loop control. For the lateral-directional aircraft dynamics in carrier landing, a linear fractional transformation gain-scheduled controller was presented in [6]. The dynamic inversion technique was used in unmanned combat aerial vehicle on an aircraft carrier in [7]. In the absence of wind and sea state turbulence, the controller performed well. After adding wind and sea state turbulence, the controller performance was degraded.

Adaptive control has become one of the most popular designs for failures and disturbances compensation. An output tracking model reference adaptive control (MRAC) scheme was developed for single-input/single-output systems in [8]. The related technical issues including design conditions, plant-model matching conditions, controller structures, adaptive laws, and stability analysis are presented in detail, with extensions to adaptive disturbance rejection. In [9], a combined direct and indirect MRAC statefeedback architecture was developed for MIMO dynamical systems with matched uncertainties and the methodology was extended to systems with a baseline controller. To solve the disturbance rejection problems, adaptive feedforward [10, 11], feedback control methods [12], terminal sliding-mode control method [13-15], and back-stepping control designs $[16,17]$ were proposed. In [18], an extension of biobjective optimal control modification for unmatched uncertain 
systems was proposed. However, the existing methods are mainly for the matched disturbance rejection, while there exists certain difficulty of achieving tracking performance, especially for the unmatched disturbances.

In this paper, an adaptive control scheme is proposed to handle wind during carrier landing. The main contributions of this paper are described as follows:

(1) With unmatched disturbance, the aircraft models in air-wake turbulence conditions during the carrier landing are analyzed. The longitudinal linearized model of a carrier-based aircraft dynamics is constructed on the final-approach.

(2) Adaptive LDU decomposition-based state-feedback controller is designed to relax design conditions, including adaptive laws and stability analysis.

(3) The proposed LDU decomposition-based disturbance rejection techniques are used to solve a typical carrier landing aircraft turbulence compensation problem. Extensive simulation results are obtained through a longitudinal aircraft dynamic model during aircraft landing.

The rest of this paper is organized as follows. In Section 2, we present the aircraft model with disturbances during the carrier landing phase. In Section 3, we propose adaptive designs to solve the aircraft disturbance compensation. We illustrate an application of the proposed adaptive design to aircraft wind disturbance rejection control. In Sections 4 and 5 some simulation results and conclusions are discussed.

\section{Longitudinal Model of Carrier-Based Aircraft on Final-Approach Dynamics in the Air Wake}

The overall carrier landing task for a fixed-wing aircraft is shown in Figure 1. The final-approach leg is typically entered from the last turn until the touchdown on the carrier deck, as illustrated in Figure 2. The turbulence is the major source of glide path and touchdown errors. In this phase, the longitudinal reference flight state is chosen as a steady rectilinear flight in air wake at a constant velocity, with constant angle of attack and a flight-path angle. The flaps and the gear are totally lowered, and two control means are employed to control the flight-path vector in the vertical plane: elevator and engine thrust. In this section, the longitudinal linear aircraft model and carrier air wake are described.

2.1. Nonlinear Aircraft Longitudinal Equations in the Calm Air. Both of the bank and sideslip angles are zero; the decoupling longitudinal of the nonlinear equations is described in the calm circumstance. The longitudinal aircraft dynamics equations are presented as follows.

The force equations are

$$
\begin{aligned}
m \dot{V} & =T \cos \alpha-D-m g \sin \gamma \\
m V \dot{\gamma} & =T \sin \alpha+L-m g \cos \gamma .
\end{aligned}
$$

The kinematic equation is

$$
\dot{\theta}=q .
$$

The moment equation is

$$
\dot{q}=\frac{M}{I_{y}} .
$$

The navigation equation is

$$
\dot{h}=V \sin \gamma .
$$

The identical equation is

$$
\gamma=\theta-\alpha
$$

2.2. Longitudinal Linearized Model of Aircraft in the Calm Air. The linear model of the longitudinal flight dynamics is constructed based on the small-perturbation equation. The linearized longitudinal flight dynamics is described as

$$
\begin{aligned}
& \dot{x}_{1}=A_{1} x_{1}+B_{1} u \\
& y_{1}=C_{1} x_{1},
\end{aligned}
$$

where $x_{1}=[v, \alpha, q, \theta, h]^{T}, u_{1}=\left[\delta_{e}, \delta_{t}\right]^{T}$, and $y_{1}=$ $[v, \alpha, q, \theta, h]^{T}$ are the system state vector, input vector, and output vector, respectively, and the matrices are

$$
A_{1}=\left[\begin{array}{ccccc}
\frac{T_{V_{0}} \cos \alpha_{0}-D_{V_{0}}}{m} & \frac{-T_{0} \sin \alpha_{0}-D_{\alpha_{0}}}{m}+g \cos \gamma_{0} & 0 & -g \cos \gamma_{0} & -\frac{D_{h_{0}}}{m} \\
-\frac{L_{V_{0}}}{m V_{0}} & \frac{-T_{0} \cos \alpha_{0}-L_{\alpha_{0}}+m g \sin \gamma_{0}}{m V_{0}} & 1 & \frac{-g \sin \gamma_{0}}{V_{0}} & -\frac{L_{h_{0}}}{m V_{0}} \\
\frac{M_{v_{0}}}{I_{y}}-\frac{M_{\dot{\alpha}_{0}}}{I_{y}}\left(\frac{L_{V_{0}}}{m V_{0}}\right) & \frac{M_{\alpha_{0}}}{I_{y}}-\frac{M_{\dot{\alpha}_{0}}}{I_{y}}\left(\frac{L_{\alpha_{0}}+T_{0} \cos \alpha_{0}}{m V_{0}}-\frac{g \sin \gamma_{0}}{V_{0}}\right) & \frac{M_{q_{0}}}{I_{y}}+\frac{M_{\dot{\alpha}_{0}}}{I_{y}}-\frac{M_{\dot{\alpha}_{0}}}{I_{y}}\left(\frac{g \sin \gamma_{0}}{V_{0}}\right) & 0 \\
0 & 0 & 1 & 0 & 0 \\
\sin \gamma_{0} & -V_{0} \cos \gamma_{0} & 0 & V_{0} \cos \gamma_{0} & 0
\end{array}\right],
$$




$$
\begin{aligned}
B_{1} & =\left[\begin{array}{ccc} 
& -\frac{D_{\delta_{e}}}{m} & -\frac{T_{\delta_{t}} \cos \alpha_{0}}{m} \\
& -\frac{L_{\delta_{e}}}{m V_{0}} & -\frac{T_{\delta_{t}} \sin \alpha_{0}}{m V_{0}} \\
\frac{M_{\delta_{e}}}{I_{y}}-\frac{M_{\dot{\alpha}_{0}}}{I_{y}}\left(\frac{L_{\delta_{e}}}{m V_{0}}\right) & -\frac{M_{\dot{\alpha}_{0}}}{I_{y}}\left(\frac{T_{\delta_{t}} \sin \alpha_{0}}{m V_{0}}\right) \\
& & 0
\end{array}\right], \\
C_{1} & =\left[\begin{array}{lllll}
1 & 0 & 0 & 0 & 0 \\
0 & 1 & 0 & 0 & 0 \\
0 & 0 & 1 & 0 & 0 \\
0 & 0 & 0 & 1 & 0 \\
0 & 0 & 0 & 0 & 1
\end{array}\right] .
\end{aligned}
$$

2.3. Longitudinal Linearized Model of Aircraft in the Carrier Air Wake. The steady component of the carrier air wake is taken into account to provide some disturbances, as a basis of our simulations.

2.3.1. Turbulence Description. The steady component of the carrier air wake is taken into account to the simulation. The superstructure and deck/hull features of an aircraft carrier are known to generate turbulent airflow behind the carrier. This region of turbulent air has become known as the burble and it is often encountered by pilots immediately after an aircraft carrier. This turbulent region of air has adverse effects on landing aircraft and can cause pilots to bolter, missing the arresting wires and requiring another landing attempt.

The burble components are determined from look-up tables scheduled on the aircraft distance behind the ship in [19-21], and the components are presented as

$$
\begin{aligned}
& \frac{u_{x}}{V_{w}} \\
& = \begin{cases}0 & X \in[-\infty,-1750) \\
\frac{0.04}{-1710+1750}(X+1750) & X \in[-1750,-1710) \\
\frac{0.02}{-1630+1710}(X+1870) & X \in[-1710,-1630) \\
\frac{-0.004}{-1550+1630}(X+430) & X \in[-1630,-1550) \\
\frac{-0.028}{-1340+1550}(X+1130) & X \in[-1550,-1340) \\
\frac{0.01}{-1160+1340}(X+1844) & X \in[-1340,-1160) \\
\frac{0.15-0.038}{-400+1160}(X+1418) & X \in[-1160,-400) \\
\frac{-0.15}{150}(X+250) & X \in[-400,-250) \\
0 & X \in[-250,0)\end{cases}
\end{aligned}
$$

$$
\begin{aligned}
& \frac{u_{y}}{V_{w}} \\
& =\left\{\begin{array}{lc}
0 & X \in[-\infty,-2650) \\
\frac{-0.076}{-2400+2650}(X+2650) & X \in[-2650,-2400) \\
\frac{-0.088+0.076}{-2200+2400}(X+3667) & X \in[-2400,-2200) \\
\frac{-0.08+0.088}{-1970+2200}(X-330) & X \in[-2200,-1970) \\
\frac{0.02+0.08}{-750+1970}(X+994) & X \in[-1970,-750) \\
\frac{-0.01-0.02}{-250+750}(X+417) & X \in[-750,-250) \\
-0.01 & X \in[-250,0) \\
X=-V \cos \gamma\left(T_{0}-t\right)-286,
\end{array}\right.
\end{aligned}
$$

where $X$ is the distance between the aircraft and the ship center of pitch, negative after of ship, $T_{0}$ is the total landing time, and $t$ is the present time.

\subsubsection{Longitudinal Linearized Model of Carrier-Based Aircraft} in Air-Wake Disturbance. The linear model of the aircraft under the air-wake disturbance is addressed in $[22,23]$. The airspeed and angle of attack are susceptible to $u_{x}$ and $u_{y}$. Because the flight speed is far larger than the wind speed, we can get

$$
\begin{aligned}
& V_{i} \approx V+u_{x} \\
& \alpha_{i} \approx \alpha+\frac{u_{y}}{V},
\end{aligned}
$$


where $V_{i}$ and $\alpha_{i}$ are the airspeed and angle of attack affected by disturbances. From (6) and (9), the linearized longitudinal dynamics of aircraft under turbulence conditions can be modeled as

$$
\dot{x}_{1}=A_{1} x_{1}+B_{1} u_{1}+B_{1 d} d(t)
$$

$$
B_{1 d}=\left[\begin{array}{cc}
\frac{T_{V_{0}} \cos \alpha_{0}-D_{V_{0}}}{m} \cos \gamma_{0} & -\frac{T \sin \alpha_{0}-D_{\alpha_{0}}}{m V_{0}} \\
\frac{T_{V_{0}} \sin \alpha_{0}+L_{V_{0}}}{m V_{0}} & \frac{T \sin \alpha_{0}+L_{\alpha_{0}}}{m V_{0}^{2}} \\
\frac{M_{\dot{\alpha}_{0}}}{I_{y}}\left(\frac{T_{V_{0}} \sin \alpha_{0}+L_{V_{0}}}{m V_{0}}\right)-\frac{M_{v_{0}}}{I_{y}} & \frac{M_{\dot{\alpha}_{0}}}{I_{y}}\left(\frac{L_{\alpha_{0}}+T_{0} \sin \alpha_{0}}{m V_{0}^{2}}\right)-\frac{M_{\alpha_{0}}}{I_{y} V_{0}} \\
0 & 0 \\
0 & 0
\end{array}\right] .
$$

\section{Adaptive Disturbance Rejection Design}

In this section, to solve turbulence compensation problem, an adaptive disturbance rejection design is developed for multivariable systems with unmatched input disturbances.

3.1. Problem Formulation. Consider the linear time-invariant system as

$$
\begin{aligned}
& \dot{x}(t)=A x(t)+B u(t)+B_{d} d(t) \\
& y(t)=C x(t),
\end{aligned}
$$

where $A \in R^{n \times n}, B \in R^{n \times M}, B_{d} \in R^{M \times p}$, and $C \in R^{M \times n}$ are constant and unknown; $x(t) \in R^{n}, u(t) \in R^{M}$, and $y(t) \in R^{M}$ are the system state vector, input vector, and output vector, respectively; $d(t)=\left[d_{1}(t), \ldots, d_{p}(t)\right]^{T} \in R^{p}$ is the disturbance vector. The disturbance signal $d(t)$ is unmatched with the control input $u(t)$, in the sense that $B$ and $B_{d}$ are not linearly dependent, $B_{d} \neq B \alpha$ for any matrix $\alpha \in R^{M \times p}$.

The control objective is to design an adaptive statefeedback control signal $u(t)$ for (12), to make closed-loop signal boundedness and the output $y(t)$ track a chosen reference signal $y_{m}(t)$ generated from a reference model:

$$
y_{m}(t)=W_{m}(s)[r](t) \in R^{M}
$$

where $W_{m}(s) \in R^{M \times M}$ is a stable transfer function matrix and $r(t) \in R^{M}$ is an external reference input signal for defining a desired $y_{m}(t)$. Note that, in this paper, we use the notation $y(t)=G(s)[u](t)$ to represent the output $y(t)$ of a system whose transfer matrix is $G(s)$ and input is $u(t)$, a convenient notation for adaptive control systems.

\subsection{Preliminaries and Assumptions}

Lemma 1 (see [8]). For any $M \times M$ strictly proper and full rank rational transfer matrix $G(s)$, there exists a lower triangular

$$
y_{1}=C_{1} x_{1}
$$

where $A_{1}, B_{1}, C_{1}, x_{1}$, and $u_{1}$ are defined in (6). The disturbance is $d(t)=\left[u_{x}, u_{y}\right]$ and the matrix $B_{1 d}$ is

polynomial matrix $\xi_{m}(s)$, defined as the left interactor matrix of $G(s)$, of the form

$$
\xi_{m}(s)=\left[\begin{array}{cccc}
d_{1}(s) & 0 & 0 & 0 \\
h_{21}^{m}(s) & d_{2}(s) & 0 & 0 \\
\vdots & \vdots & \vdots & \vdots \\
h_{M 1}^{m}(s) & h_{M 2}^{m}(s) & \cdots & d_{M}(s)
\end{array}\right]
$$

where $h_{i j}^{m}(s), j=1,2, \ldots, M-1, i=2, \ldots, M$, are some polynomials and $d_{i}(s)$ are any chosen monic stable polynomials such that the high-frequency gain matrix of $G(s)$ defined as $K_{p}=\lim _{s \rightarrow \infty} \xi_{m}(s) G(s)$.

Lemma 2 (see [24]). Every $M \times M$ real matrix $K_{p}$ with nonzero leading principal minors $\Delta_{1}, \Delta_{2}, \ldots, \Delta_{M}$ can be uniquely factored as

$$
K_{p}=L D U,
$$

where $L \in R^{M \times M}$ is unity lower triangular, $U \in R^{M \times M}$ is unity upper triangular, and

$$
\begin{aligned}
D & =\operatorname{diag}\left\{d_{1}, d_{2}, \ldots, d_{m}\right\} \\
& =\operatorname{diag}\left\{\Delta_{1}, \Delta_{2} \Delta^{-1}, \ldots, \Delta_{m} \Delta_{m-1}^{-1}\right\} .
\end{aligned}
$$

Assumption 3. All zeros of $G_{0}(s)=C(s I-A)^{-1} B$ are stabilizable and detectable.

Assumption 4. $G_{0}(s)$ is strictly proper with full rank and has a known modified interactor matrix $\xi_{m}(s)$ such that $K_{p}=$ $\lim _{s \rightarrow \infty} \xi_{m}(s) G_{0}(s)$ is finite and nonsingular (so that $W_{m}(s)=$ $\xi_{m}^{-1}(s)$ can be chosen as the transfer matrix for the reference model system). 


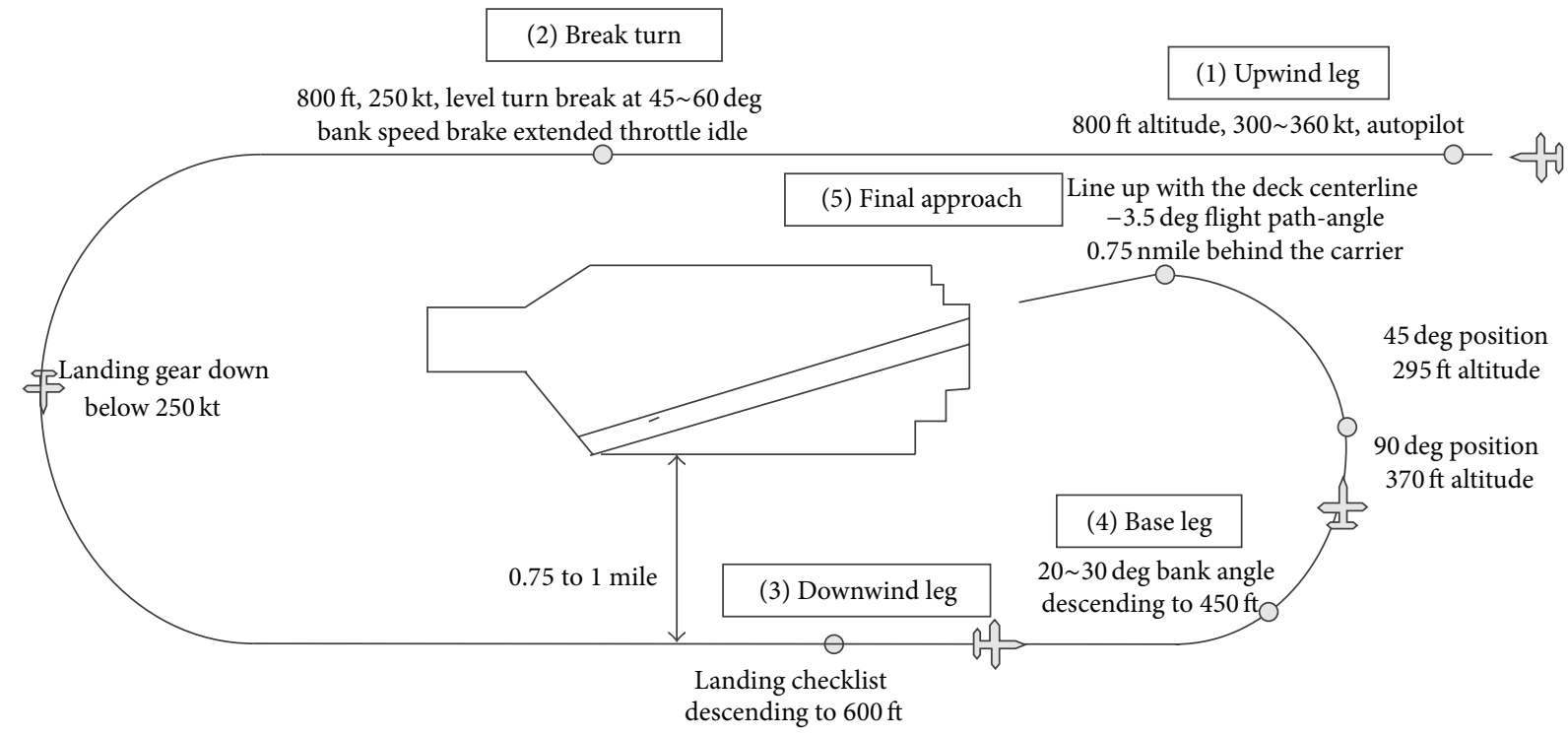

FIGURE 1: Procedures of carrier landing for the aircraft.

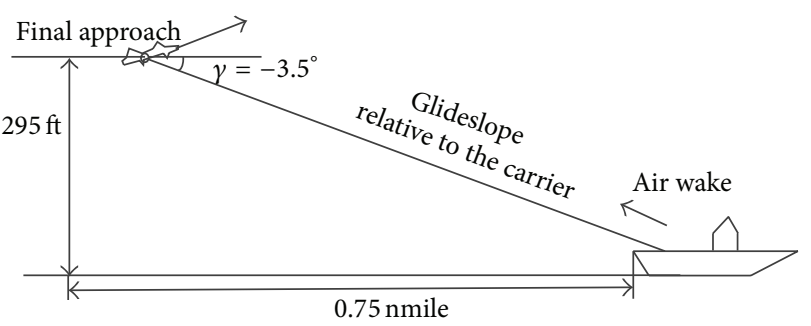

FIgURE 2: Final carrier landing phase.

Assumption 5. The leading principal minors of the highfrequency gain matrix $K_{p}$ are nonzero, and their signs are known.

From plant (12), the control and disturbance transfer functions are obtained as $G_{0}(s)=C(s I-A)^{-1} B$ and $G_{d}(s)=C(s I-A)^{-1} B_{d}$ and are expressed in their left coprime polynomial matrix decompositions: $G_{0}(s)=P_{l}^{-1}(s) Z_{0}(s)$ and $G_{d}(s)=P_{l}^{-1}(s) Z_{d}(s)$, where $P_{l}(s), Z_{0}(s) \in R^{M \times M}$ and $Z_{d}(s) \in$ $R^{M \times p}$ are some polynomial matrices.

Assumption 6. The transfer matrix $Z_{0}^{-1}(s) Z_{d}(s)$ is proper.

Remark 7. Assumption 3 is for output matching and internal signal stability. Assumption 4 is for choosing the reference system model for adaptive control. Assumption 5 is for designing adaptive parameter update laws. Assumption 6 is the relative degree condition from the control input $u(t)$ and the disturbance input $d(t)$ to the output $y(t)$ for the design of a derivative-free disturbance rejection scheme.

3.3. Nominal Disturbance Rejection Design. With the knowledge of the plant and disturbance parameters, the nominal state-feedback controller is

$$
u^{*}(t)=K_{1}^{* T} x(t)+K_{2}^{*} r(t)+K_{3}^{*}(t),
$$

where the nominal parameters $K_{1}^{* T} \in R^{M \times n}$ and $K_{2}^{* T} \epsilon$ $R^{M \times M}$ are for the plant-model output matching, and $K_{3}^{*}(t) \epsilon$ $R^{M}$ is used to cancel the effect of the disturbance $d(t)$.

Lemma 8 (see [25]). The matrix $K_{d}=\lim _{s \rightarrow \infty} \xi_{m}(s) G_{d}(s)$ is finite if $Z_{0}^{-1}(s) Z_{d}(s)$ is proper.

Based on Lemma 8, the existence of a nominal controller (17) is established a follows.

Theorem 9. For plant (12) with the unmatched disturbances, under Assumptions 3 and 6, there exists a state-feedback control law, to make the boundedness of all closed-loop signals, disturbance rejection, and output tracking of the reference $y_{m}(t)$.

From plant (12), the input-output form is obtained as

$$
y(t)=G_{0}(s)[u](t)+\bar{y}(t),
$$

with $\bar{y}(t)=G_{0}(s)[d](t)=C(s I-A)^{-1} B_{d}[d](t)$.

Operate the interactor matrix (a polynomial matrix) $\xi_{m}(s)$ on plant (12), $\dot{x}(t)=A x(t)+B u(t)+B_{d} d(t), y(t)=$ $C x(t)$, to reach an expression of $\xi_{m}(s)\left[y_{m}\right](t)$ in a possible form:

$$
\begin{aligned}
\xi_{m}(s)[y](t)= & -\bar{K}_{0} x(t)+\bar{K}_{p} u(t)+\bar{K}_{p 1} \dot{u}+\cdots \\
& +\bar{K}_{p l 0} u^{\left(l_{0}\right)}(t)+\bar{K}_{d} d(t)+\bar{K}_{d 1} \dot{d}(t) \\
& +\cdots+\bar{K}_{d l 1} d^{(l 1)}(t),
\end{aligned}
$$

with some constant matrices $\bar{K}_{0} \in R^{M \times n}, \bar{K}_{p} \in R^{M \times M}, \bar{K}_{p j} \in$ $R^{M \times M}, j=1,2, \ldots, l_{0}, \bar{K}_{d} \in R^{M \times p}$, and $\bar{K}_{d i} \in R^{M \times p}, i=$ $1,2, \ldots, l_{1}$, for some integers $l_{0}, l_{1} \geq 0$. From (12) and (18), we have

$$
x(s)=(s I-A)^{-1} B u(s)+(s I-A)^{-1} B_{d} d(s) .
$$


Expressing (19) in $s$ domain and using (20), we have

$$
\begin{aligned}
\xi_{m}(s) y(s)= & -\bar{K}_{0}(s I-A)^{-1} B u(s)+\bar{K}_{p} u(s) \\
& +\bar{K}_{p 1} s u(s)+\cdots+\bar{K}_{p l_{0}} s^{l_{0}} u(s) \\
& -\bar{K}_{0}(s I-A)^{-1} B_{d} d(s)+\bar{K}_{d} d(s) \\
& +\bar{K}_{d 1} s d(s)+\cdots+\bar{K}_{d l_{1}} s^{l_{1}} d(s) .
\end{aligned}
$$

From Assumption 4, $K_{p}=\lim _{s \rightarrow \infty} \xi_{m}(s) G_{0}(s)$ is finite and nonsingular and from Assumption $6, K_{p j}=0, j=$ $1, \ldots, l_{0}, \bar{K}_{P}=K_{p}$, and $K_{d j}=0, j=1, \ldots, l_{0}, \bar{K}_{d}=K_{d}$. Hence, we have

$$
\xi_{m}(s)[y](t)=-\bar{K}_{0} x(t)+K_{p} u(t)+K_{d} d(t) .
$$

From (17), the control law can be designed as

$$
u(t)=u^{*}(t)=K_{1}^{* T} x(t)+K_{2}^{*} r(t)+K_{3}^{*}(t),
$$

where $K_{1}^{* T}=K_{p}^{-1} \bar{K}_{0}, K_{2}^{*}=K_{p}^{-1}$, and $K_{3}^{*}(t)=K_{3 d} d(t)$ with $K_{3 d}(t)=-K_{p}^{-1} K_{d}$, which leads to the output matching: $\xi_{m}(s)[y](t)=r(t)$. We applied (23) to plant (12); we have

$$
\begin{aligned}
y(t)= & C\left(s I-A-B K_{1}^{* T}\right)^{-1} B K_{2}^{*}[r](t) \\
& +C\left(s I-A-B K_{1}^{* T}\right)^{-1} B\left[K_{3}^{*}\right](t) \\
& +C\left(s I-A-B K_{1}^{* T}\right)^{-1} B_{d} d(s) \\
= & W_{m}(s)[r](t)=y_{m}(t) .
\end{aligned}
$$

Remark 10. From (24), we can conclude that the plant-model matching conditions are

$$
\begin{aligned}
C\left(s I-A-B K_{1}^{* T}\right)^{-1} B K_{2}^{*} \\
=W_{m}(s) W_{m}(s) K_{2}^{*-1} K_{3}^{*}(s) \\
\quad+C\left(s I-A-B K_{1}^{* T}\right)^{-1} B_{d} d(s)=0 .
\end{aligned}
$$

For the plant-model matching condition (25), there exist constant matrices $K_{1}^{* T}$ and $K_{2}^{*}$ and $K_{3}^{*}(t)$, if and only if the relative degree condition in Assumption 6 is satisfied.

3.4. Parameterizations of the Term $K_{3}^{*}(t)$. For the disturbance vector $d(t) \in R^{p}$, each element $d_{j}(t)$ in (12) can be expressed as

$$
d_{j}(t)=d_{j 0}+\sum_{k=1}^{q_{j}} d_{j k} f_{j k}(t)=\mu_{j}^{* T} f_{j}(t),
$$

$$
j=1,2, \ldots, p
$$

with some unknown constants $d_{j 0}, d_{j k}$ and some known bounded continuous signals $f_{j k}, j=1,2, \ldots, p, k=$ $1,2, \ldots, q_{j}$.
Remark 11. For model (26), if $q_{j}=0$, then $d_{j}(t)=d_{j 0}, j=$ $1,2, \ldots, p$, representing a constant disturbance signal. If we choose $q_{j}=1$ and $f_{j 1}(t)=\sin \left(w_{j 1} t\right)$, then $d_{j}(t)=$ $d_{j 0}+d_{j 1} \sin \left(w_{j 1} t\right), j=1,2, \ldots, p$, representing time-variant period disturbances. A large class of practical disturbances in control applications can be approximated by a proper selection of this basis function $f_{j k}(t)$ in (26).

The parameter matrix and the disturbance signal components are

$$
\begin{aligned}
& \mu_{j}^{*}=\left[d_{j 0}, d_{j 1}, \ldots, d_{j q j}\right]^{T} \in R^{q_{j}+1}, \\
& f_{j}(t)=\left[1, f_{j 1}(t), \ldots, f_{j q j}(t)\right]^{T} \in R^{q_{j}+1}, \\
& \quad j=1,2, \ldots, p .
\end{aligned}
$$

Hence, the disturbance $d(t)$ is expressed as

$$
d(t)=N^{* T} f(t)
$$

$$
\begin{gathered}
N^{* T}=\left[\begin{array}{ccccc}
\mu_{1}^{T} & 0_{\left(q_{2}+1\right)}^{T} & 0_{\left(q_{3}+1\right)}^{T} & \cdots & 0_{\left(q_{p}+1\right)}^{T} \\
0_{\left(q_{1}+1\right)}^{T} & \mu_{2}^{* T} & 0_{\left(q_{3}+1\right)}^{T} & \cdots & 0_{\left(q_{p}+1\right)}^{T} \\
\vdots & \vdots & \vdots & \vdots & \vdots \\
0_{\left(q_{1}+1\right)}^{T} & 0_{\left(q_{2}+1\right)}^{T} & 0_{\left(q_{3}+1\right)}^{T} & \cdots & \mu_{p}^{* T}
\end{array}\right] \in R^{p \times q}, \\
f(t)=\left[\begin{array}{llll}
f_{1}^{T}(t) & f_{2}^{T}(t) & \cdots & f_{p}^{T}(t)
\end{array}\right]^{T} \in R^{q} \\
q=q_{1}+q_{2}+\cdots+q_{p}+p .
\end{gathered}
$$

With $K_{3 d}^{*}=\left[k_{3 d 1}^{*}, k_{3 d 2}^{*}, \ldots, k_{3 d p}^{*}\right], k_{3 d j}^{*} \in R^{M}, j=$ $1,2, \ldots, p$, the disturbance rejection term $K_{3}^{*}(t)$ is parameterized as

$$
K_{3}^{*}(t)=K_{3 d}^{*} d(t)=K_{3 d}^{*} N^{* T} f(t)=\Phi_{3}^{*} f(t),
$$

where the parameter matrix is

$$
\begin{aligned}
& \Phi_{3}^{*}=\left[\phi_{31}^{*}, \phi_{32}^{*}, \ldots, \phi_{3 p}^{*}\right] \in R^{M \times q}, \\
& \qquad=q_{1}+q_{2}+\cdots+q_{p}+p, \\
& \phi_{3 j}^{*}=k_{3 d j}^{*} \mu_{j}^{* T} \in R^{M \times\left(q_{j}+1\right)}, \quad j=1,2, \ldots, p .
\end{aligned}
$$

Next, the adaptive disturbance rejection design will be studied for the plant with uncertainties from the plant and unmatched disturbances.

3.5. Error Equation. Applying (23) to plant (12), the closedloop system becomes

$$
\begin{aligned}
\dot{x}(t)= & \left(A+B K_{1}^{* T}\right) x(t)+B K_{2}^{*} r(t)+B K_{3}^{*}(t) \\
& +B_{d} d(t) \\
& +B\left[u(t)-K_{1}^{* T} x(t)-K_{2}^{*} r(t)-K_{3}^{*}(t)\right] y(t) \\
= & C x(t) .
\end{aligned}
$$


In view of (24), (25), and (31), the output tracking error equation is

$$
\begin{aligned}
e(t)= & y(t)-y_{m}(t) \\
= & W_{m}(s) K_{p}^{*}\left[u-K_{1}^{* T} x-K_{2}^{*} r-K_{3}^{*}\right](t) \\
& +f_{p}(t), \quad K_{p}^{*}=K_{2}^{*-1},
\end{aligned}
$$

where $f_{p}(t)=C e^{\left(A+B K_{1}^{* T}\right) t} x(0)$ converges to zero exponentially fast due to the stability of $A+B K_{1}^{* T}$ and $W_{m}(s)=\xi_{m}^{-1}(s)$. Hence, we have

$$
\begin{aligned}
\xi_{m} & (s)[e](t) \\
\quad= & K_{p}\left[u(t)-K_{1}^{* T} x(t)-K_{2}^{*} r(t)-K_{3}^{*}(t)\right] .
\end{aligned}
$$

To deal with the uncertainty of the high-frequency gain matrix $K_{p}$, the LDU decomposition of $K_{p}$ is used in (33), so that we have

$$
\begin{aligned}
& L^{-1} \xi_{m}(s)[e](t) \\
& \quad=D U\left(u(t)-K_{1}^{* T} x(t)-K_{2}^{*} r(t)-K_{3}^{*}(t)\right) .
\end{aligned}
$$

We have the following equation:

$$
U u(t)=u(t)-(I-U) u(t) .
$$

With (34) and (35), we have

$$
\begin{gathered}
L^{-1} \xi_{m}(s)[e](t)=D[u(t)-(I-U) u(t) \\
\left.-U\left[K_{1}^{* T} x(t)-K_{2}^{*} r(t)-K_{3}^{*}(t)\right]\right] .
\end{gathered}
$$

The new equation is

$$
L^{-1} \xi_{m}(s)[e](t)=D\left[u(t)-\Phi_{0}^{*} u(t)-\Phi_{1}^{* T} \omega(t)\right],
$$

where $\Phi_{1}^{* T}=\left[U K_{1}^{* T}, U K_{2}^{*}, U K_{3 f}^{*}\right]$ and $\omega(t)=\left[x^{T}(t), r^{T}(t)\right.$, $\left.f^{T}(t)\right]^{T}$. This new equation has a new controller structure:

$$
u(t)=\Phi_{0} u(t)+\Phi_{1}^{T} \omega(t),
$$

where $\Phi_{0}$ and $\Phi_{1}^{T}$ are the estimates of $\Phi_{0}^{*}$ and $\Phi_{1}^{* T}$ and $\Phi_{0}$ is upper triangular with zero diagonal elements (only its nonzero elements are estimated). The matrix $\Phi_{0}$ has the same strictly form as that of $\Phi_{0}^{*}=(I-U)$ :

$$
\Phi_{0}=\left[\begin{array}{ccccc}
0 & \phi_{12} & \phi_{13} & \cdots & \phi_{1 M} \\
0 & 0 & \phi_{23} & \cdots & \phi_{2 M} \\
\vdots & \vdots & \vdots & \vdots & \\
0 & 0 & \cdots & 0 & \phi_{M-1 M} \\
0 & 0 & \cdots & 0 & 0
\end{array}\right] \in R^{M \times M} .
$$

From (37) and (38), we obtain a new error model:

$$
\xi_{m}(s)[e](t)+\Theta_{0}^{*} \xi_{m}(s)[e](t)=D \widetilde{\Phi}^{T}(t) \bar{\omega}(t),
$$

where the parameter error is $\widetilde{\Phi}(t)=\Phi(t)-\Phi^{*}$ and $\Phi^{T}(t)=$ $\left[\Phi_{0}(t), \Phi_{1}^{T}(t)\right]$ is the estimate of unknown parameter matrix $\Phi^{* T}=\left[\Phi_{0}^{*}, \Phi_{1}^{* T}\right], \bar{\omega}(t)=\left[u^{T}(t), \omega^{T}(t)\right]^{T}$, and $\omega(t)=$ $\left[x^{T}(t), r^{T}(t), f^{T}(t)\right]^{T}$, where $\Theta_{0}^{*}=\left(L^{-1}-I\right)$ is introduced to parameterize the unknown matrix $L$, which has a special form:

$$
\Theta_{0}^{*}=\left[\begin{array}{cccc}
0 & 0 & \cdots & 0 \\
\theta_{21}^{*} & 0 & \cdots & 0 \\
\theta_{31}^{*} & \theta_{32}^{*} & \cdots & 0 \\
\vdots & \vdots & \vdots & 0 \\
\theta_{M-11}^{*} & \cdots & 0 & 0 \\
\theta_{M 1}^{*} & \cdots & \theta_{M M-1}^{*} & 0
\end{array}\right] \in R^{M \times M}
$$

For such a matrix $\Theta_{0}^{*}$, the parameter vectors are defined as

$$
\begin{gathered}
\theta_{2}^{*}=\theta_{21}^{*} \in R, \\
\theta_{3}^{*}=\left[\theta_{31}^{*}, \theta_{32}^{*}\right]^{T} \in R^{2},
\end{gathered}
$$

$$
\begin{aligned}
\theta_{M-1}^{*} & =\left[\theta_{M-11}^{*}, \ldots, \theta_{M-1 M-2}^{*}\right] \in R^{M-2}, \\
\theta_{M}^{*} & =\left[\theta_{M 1}^{*}, \ldots, \theta_{M M-2}^{*}\right] \in R^{M-1},
\end{aligned}
$$

and their estimates are

$$
\begin{gathered}
\theta_{2}(t)=\theta_{21}(t) \in R, \\
\theta_{3}(t)=\left[\theta_{31}(t), \theta_{32}(t)\right]^{T} \in R^{2},
\end{gathered}
$$

$$
\begin{gathered}
\theta_{M-1}(t)=\left[\theta_{M-11}(t), \ldots, \theta_{M-1 M-2}(t)\right] \in R^{M-2}, \\
\theta_{M}(t)=\left[\theta_{M 1}(t), \ldots, \theta_{M M-2}(t)\right] \in R^{M-1} .
\end{gathered}
$$

We introduce a filter $h(s)=1 / f(s)$, where $f(s)$ is chosen as a stable and monic polynomial whose degree is equal to the maximum degree of the modified interactor $\xi_{m}(s)$. Operating both sides of (40) by $h(s) I_{M}$ leads to

$$
\begin{gathered}
\xi_{m}(s) h(s)[e](t)+\Theta_{0}^{*} \xi_{m}(s) h(s)[e](t) \\
=\operatorname{Dh}(s)\left[\widetilde{\Phi}^{T} \bar{\omega}\right](t) .
\end{gathered}
$$

We define

$$
\begin{aligned}
\bar{e}(t) & =\xi_{m}(s) h(s)[e](t)=\left[\bar{e}_{1}(t), \ldots, \bar{e}_{M}(t)\right]^{T}, \\
\eta_{i}(t) & =\left[\bar{e}_{1}(t), \ldots, \bar{e}_{i-1}(t)\right]^{T} \in R^{i-1}, \\
& i=2,3, \ldots, M .
\end{aligned}
$$

From (45) and (46) in (44), we obtained

$$
\begin{aligned}
\bar{e}(t) & +\left[0, \theta_{2}^{* T} \eta_{2}(t), \theta_{3}^{* T} \eta_{3}(t), \ldots, \theta_{M}^{* T} \eta_{M}(t)\right]^{T} \\
= & D h(s)\left[\widetilde{\Phi}^{T} \bar{\omega}\right](t) .
\end{aligned}
$$


Based on the parameterized error equation (47), an estimation error signal is introduced:

$$
\begin{aligned}
\varepsilon(t)= & \bar{e}(t)+\left[0, \theta_{2}^{T} \eta_{2}(t), \theta_{3}^{T} \eta_{3}(t), \ldots, \theta_{M}^{T} \eta_{M}(t)\right]^{T} \\
& +\Psi(t) \xi(t) \in R^{M},
\end{aligned}
$$

where $\Psi(t) \in R^{M \times M}$ is the estimate of $\Psi^{*}=D$ and

$$
\begin{aligned}
& \zeta(t)=h(s)[\bar{\omega}](t), \quad i=1,2, \ldots, M, \\
& \xi(t)=\Phi^{T}(t) \zeta(t)-h(s)\left[\Phi^{T} \bar{\omega}\right](t),
\end{aligned}
$$

$$
i=1,2, \ldots, M \text {. }
$$

It then follows from (44) (47) and (48) that

$$
\begin{aligned}
\varepsilon(t)= & {\left[0, \widetilde{\theta}_{2}^{T} \eta_{2}(t), \widetilde{\theta}_{3}^{T} \eta_{3}(t), \ldots, \widetilde{\theta}_{M}^{T} \eta_{M}(t)\right]^{T}+D \widetilde{\Phi}^{T} } \\
& +\widetilde{\Psi}(t) \xi(t),
\end{aligned}
$$

where $\widetilde{\Psi}(t)=\Psi(t)-\Psi^{*}$ and $\widetilde{\theta}_{i}=\theta_{i}-\theta_{i}^{*}$ are the parameter errors. This error model is choice for update laws.

3.6. Adaptive Laws. Based on the error model (50), the adaptive laws are chosen as

$$
\begin{aligned}
\dot{\theta}_{i}(t) & =-\frac{\Gamma_{\theta_{i}} \varepsilon_{i}(t) \eta_{i}(t)}{m^{2}(t)}, \quad i=2, \ldots, M, \\
\dot{\Phi}^{T}(t) & =-\frac{D \varepsilon(t) \zeta^{T}(t)}{m^{2}(t)}, \\
\dot{\Psi}(t) & =-\frac{\Gamma \varepsilon(t) \xi^{T}(t)}{m^{2}(t)},
\end{aligned}
$$

where $\Gamma_{\theta_{i}}=\Gamma_{\theta_{i}}^{T}>0, i=2,3, \ldots, M$, and $\Gamma=$ $\Gamma^{T}>0$ are adaptive gains; $D$ is defined in $(15) ; \varepsilon(t)=$ $\left[\varepsilon_{1}(t), \varepsilon_{2}(t), \ldots, \varepsilon_{M}(t)\right]^{T}$ is calculated from (50); and

$$
m^{2}(t)=1+\zeta^{T}(t) \zeta(t)+\xi^{T}(t) \xi(t)+\sum_{i=2}^{M} \eta_{i}^{T} \eta_{i}(t) .
$$

3.7. Stability Analysis. To analyze the closed-loop system stability, we first establish some desired properties of the adaptive parameter update laws mentioned above.

\section{Lemma 12 (see [8]). The adaptive laws ensure that}

(i) $\theta_{i}(t) \in L^{\infty}, i=2,3, \ldots, M, \Phi(t) \in L^{\infty}, \Psi(t) \in L^{\infty}$, and $\varepsilon(t) / m(t) \in L^{2} \cap L^{\infty}$

(ii) $\dot{\theta}_{i}(t) \in L^{2} \cap L^{\infty}, i=2,3, \ldots, M, \dot{\Phi}(t) \in L^{2} \cap L^{\infty}$, and $\dot{\Psi}(t) \in L^{2} \cap L^{\infty}$.

Based on Lemma 12, the following desired closed-loop system properties are established.

Theorem 13. For plant (12) with uncertainties from the system parameters and disturbance (26) under Assumptions 3-6, and the reference model (13), the LDU decomposition-based MRAC scheme with the adaptive controller (38) and adaptive parameter update laws (51) guarantees closed-loop system boundedness and asymptotic output tracking $\lim _{t \rightarrow \infty} e(t)=0$ with $e(t)=y(t)-y_{m}(t)$.

Proof (outline). The proof of this stability theorem can be established through using a unified framework. Because the control input $u(t)$ described in (38) depends on the state $x(t)$, it first needs to be expressed by using the system output $y(t)$ through establishing the state observer of the plant:

$$
\dot{\hat{x}}(t)=(A-L C) \dot{\hat{x}}(t)+B u(t)+B_{d} d(t)+L y(t),
$$

where $L \in R^{n \times M}$ is a gain matrix such that $A-L C$ is stable, which is possible, and $(A C)$ is assumed to be detectable. Hence, we have

$$
\begin{aligned}
u(t)= & \Phi_{1}^{T}(t) \omega_{1}(t)+\Phi_{2}^{T}(t) \omega_{2}(t)+\Phi_{3 d}^{T}(t) \omega_{3}(t) \\
& +K_{2}(t) r(t)+\Phi_{3}(t) f(t),
\end{aligned}
$$

where $\Phi_{1}^{T}(t), \Phi_{2}^{T}(t), \Phi_{3 d}^{T}(t), K_{2}(t)$, and $\Phi_{3}(t)$ are adaptive estimates of the corresponding nominal controller parameters and

$$
\begin{aligned}
& \omega_{1}(t)=\frac{a(s)}{\Lambda(s)}[u](t), \\
& \omega_{2}(t)=\frac{a(s)}{\Lambda(s)}[y](t), \\
& \omega_{3}(t)=\frac{b(s)}{\Lambda(s)}[f](t),
\end{aligned}
$$

with $a(s)=\left[\begin{array}{llll}I_{M} & s I_{M} & \cdots & s^{n-1} I_{M}\end{array}\right]^{T}, b(s)=$ $\left[\begin{array}{llll}I_{q} & s I_{q} & \cdots & s^{n-1} I_{q}\end{array}\right]^{T}$, and $\Lambda(s)$ being a chosen monic stable polynomial of degree $n$, which has the same eigenvalues with $A-L C$. Then, introducing the fictitious filters for the plant $y(t)=C(s I-A)^{-1} B u(t)+C(s I-A)^{-1} B_{d} d(t)$ and using series transformations, the control input described as in (54) is transformed into the form

$$
\begin{aligned}
u(t)= & G_{11}(s, \cdot)[\bar{y}](t)+G_{12}(s, \cdot)[r](t) \\
& +G_{13}(s, \cdot)[f](t)+G_{14}(s, \cdot)\left[f_{p}\right](t),
\end{aligned}
$$

where $\bar{y}(t)=h(s)[y](t)(h(s)$ is given below (43)) and $G_{11}(s, \cdot), G_{12}(s, \cdot), G_{13}(s, \cdot)$, and $G_{14}(s, \cdot)$ are proper stable operators with finite gains. Furthermore, a filtered version of the output signal $y(t)$ is expressed in a feedback framework:

$$
\begin{gathered}
\|\bar{y}(t)\| \leq x_{0}+\beta_{1} \int_{0}^{t} e^{-\alpha_{1}(t-\tau)} x_{1}(\tau) \\
\cdot\left(\int_{0}^{\tau} e^{-\alpha_{2}(\tau-\omega)}\|\bar{y}(\bar{\omega})\| d \bar{\omega}\right) d \tau,
\end{gathered}
$$


for some $\beta_{1}, \alpha_{1}, \alpha_{2}>0$, and $x_{1}(t)=\|\dot{\Phi}(t)\|+\|\varepsilon(t)\| m(t) \epsilon$ $L^{2} \cap L^{\infty}$. Applying the small gain lemma to (57), we conclude that $\bar{y}(t) \in L^{\infty}$, and so $y(t), u(t) \in L^{\infty}$. Thus, the signals satisfy $\bar{\omega}(t), \zeta(t), \xi(t), m(t), \varepsilon \in L^{\infty}$. Furthermore, $\theta_{i}, \varepsilon(t) / m(t), \dot{\Phi}(t), \dot{\Psi}(t) \in L^{2}$ (Lemma 12) are satisfied, and in turn $\xi(t)$ and $e(t)=y(t)-y_{m}(t)$, such that $e(t)=y(t)-y_{m}(t)$ converges to zero.

\section{Simulation Study}

4.1. Aircraft Model in Turbulence. The proposed multivariable adaptive disturbance rejection scheme is applied to a carrier landing system using LDU based decomposition. The aircraft longitudinal model defined in planted (6) is derived in [26]. The system parameter matrices are described as

$$
\begin{aligned}
& A=\left[\begin{array}{ccccc}
-0.03858 & 18.984 & 0 & -32.139 & 1.3233 E-4 \\
-0.001028 & -0.63253 & 1 & 0.005612 & 3.7553 E-6 \\
7.8601 E-5 & -0.75905 & -0.5183 & -0.0079341 & -3.0808 E-7 \\
0 & 0 & 1 & 0 & 0 \\
-0.04362 & -249.76 & 0 & 249.76 & 0
\end{array}\right] \text {, } \\
& B=\left[\begin{array}{cc}
10.1 & 0 \\
-1.5446 E-4 & 0 \\
0.024656 & -0.01077 \\
0 & 0 \\
0 & 0
\end{array}\right] \\
& C=\left[\begin{array}{lllll}
1 & 0 & 0 & 0 & 0 \\
0 & 0 & 0 & 0 & 1
\end{array}\right] \text {. }
\end{aligned}
$$

The turbulence disturbances are described in [22, 23]; we can get

$$
\begin{aligned}
B_{d} & =\left[\begin{array}{llll}
B_{d 1}, B_{d 2}
\end{array}\right], \\
B_{d 1} & =\left[\begin{array}{lllll}
-0.0386 & 0.001 & 7.8601 E-5 & 0 & 0
\end{array}\right]^{T}, \\
B_{d 2} & =\left[\begin{array}{lllll}
-0.05262 & 0.00251 & -0.00304 & 0 & 0
\end{array}\right]^{T} .
\end{aligned}
$$

4.2. Adaptive Control Design. For the aircraft system, the transfer function, $G_{0}(s)=C(s I-A)^{-1} B$, has stable zeros, $s_{1}=-4.507, s_{2}=-0.91$, and $s_{3}=-0.5685$, and is strictly proper and full rank. The interactor matrix is chosen as

$$
\xi_{m}(s)=\operatorname{diag}\left\{s+1(s+1)^{2}\right\} .
$$

The high-frequency matrix is

$$
K_{p}=\lim _{s \rightarrow \infty} \xi_{m}(s) G_{0}(s)=\left[\begin{array}{cc}
2.2673 & 8.8455 \\
-0.0902 & -0.3521
\end{array}\right] \text {, }
$$

and it is is finite and nonsingular and the matrix

$$
K_{d}=\lim _{s \rightarrow \infty} \xi_{m}(s) G_{d}(s)=\left[\begin{array}{rr}
-0.0386 & -0.0526 \\
-0.2481 & -0.6246
\end{array}\right]
$$

is finite. From the specified left coprime polynomial matrix decompositions, $G_{0}(s)=P_{l}^{-1}(s) Z_{0}(s)$ and $G_{d}(s)=$ $P_{l}^{-1}(s) Z_{d}(s)$, we can obtain

$$
\lim _{s \rightarrow \infty} Z_{0}^{-1}(s) Z_{d}(s)=\left[\begin{array}{cc}
-0.017 & -0.0064 \\
2.7493 & 1.7739
\end{array}\right],
$$

which means that the relative degree condition in Assumption 6 can be ensured.

The related gain parameters in adaptive laws (51) are chosen as $\Gamma_{\theta}=100, D=\operatorname{diag}\{2 \quad 2\}$, and $\Gamma=\operatorname{diag}\left\{\begin{array}{ll}10 & 10\end{array}\right.$.

4.3. Simulation Results. For this simulations study, the initial state is chosen as $x_{0}(t)=\left[\begin{array}{lllll}240 & 0 & 0 & 0 & 295\end{array}\right]$, and the initial controller parameters are set as $70 \%$ of their true values. As shown in Figures 3 and 4, the LDU based adaptive controller can ensure that the aircraft system output signal tracks the reference height tightly. Figures 5 and 6 show tracking performances of the automatic carrier landing system where the adaptive controller is used under the final-approach leg. Figure 7 shows the surface deflections and power control, when the aircraft receives a time varying turbulence. From the simulations, the automatic carrier landing system with the proposed adaptive controller is well performed in the turbulence. This indicates the disturbance adaptive controller can be used in carrier air-wake in the final-approach air condition. 


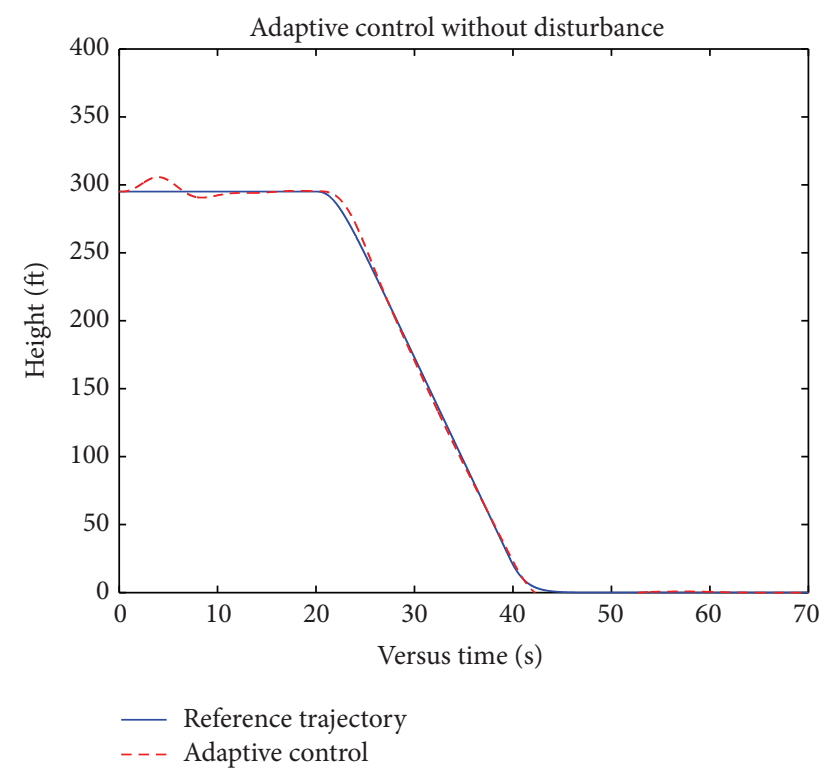

FIGURE 3: Final landing phase altitude for the aircraft.

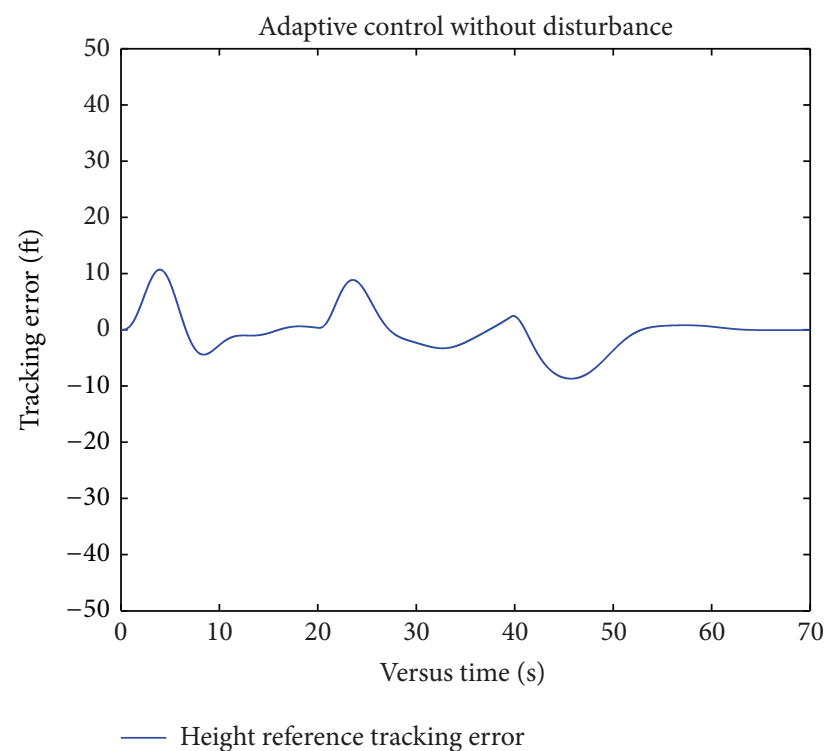

FIGURE 4: Final landing phase tracking error for the aircraft.

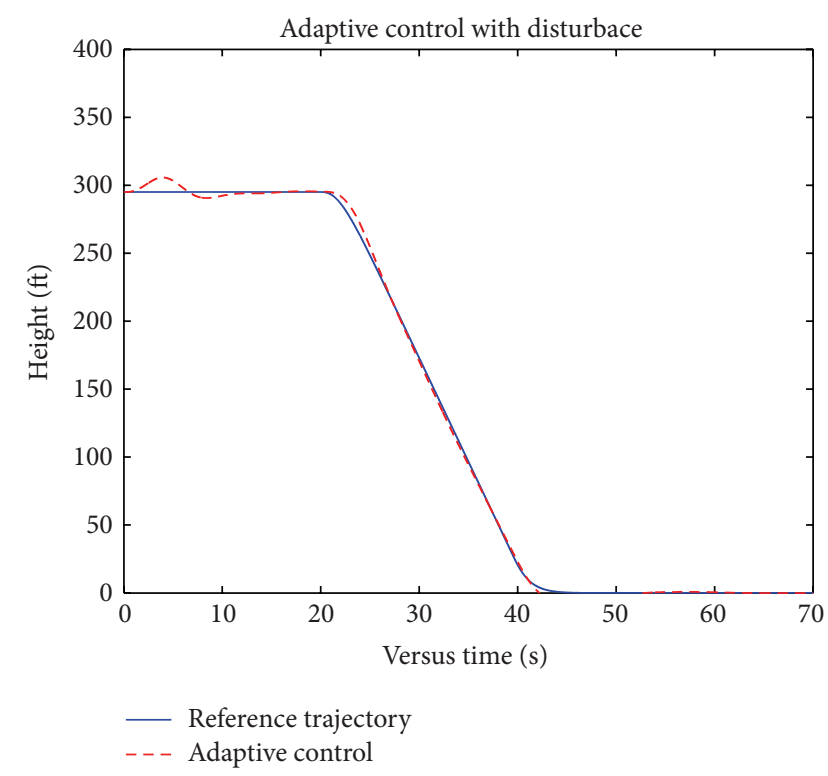

FIgURE 5: Final landing phase altitude for the aircraft.

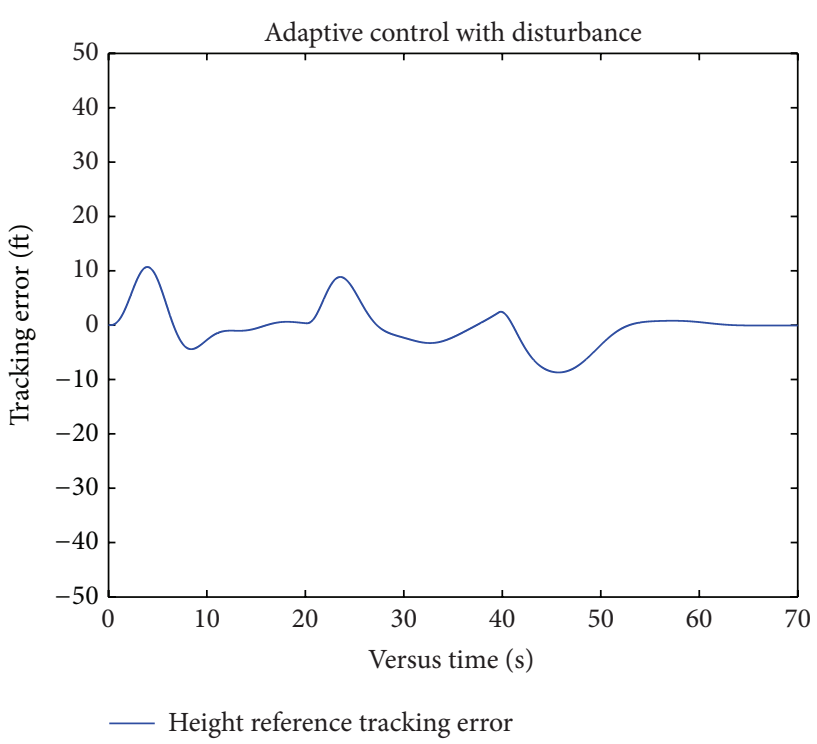

FIGURE 6: Final landing phase tracking error for the aircraft.

\section{Conclusions}

In this paper, a multivariable disturbance rejection scheme is presented to solve the wind turbulence problem. The statefeedback output tracking MRAC scheme is designed based on the LDU decomposition of the high-frequency gain matrix. The aircraft carrier landing system under aircraft carrier air wake is analyzed. The proposed LDU decomposition-based disturbance rejection techniques are used to solve a typical carrier landing aircraft turbulence compensation problem. Finally, simulation results have been presented to show that MRAC-based disturbance rejection scheme is an effective method of the carrier landing system with the disturbances.

\section{Nomenclature}

$D_{\delta_{e}}: \quad$ Aerodynamic drag derivative with respect to elevator deflection angle

$T, D, L$ : Engine thrust, aerodynamic drag force, and aerodynamic lift force

$\alpha, \theta, \gamma$ : Angle of attack, pitch, and flight-path slope

$h$ : $\quad$ Altitude of aircraft

g: Gravitational acceleration

$m: \quad$ Mass of aircraft

$V: \quad$ Airspeed of aircraft

$q$ : $\quad$ Pith rate 

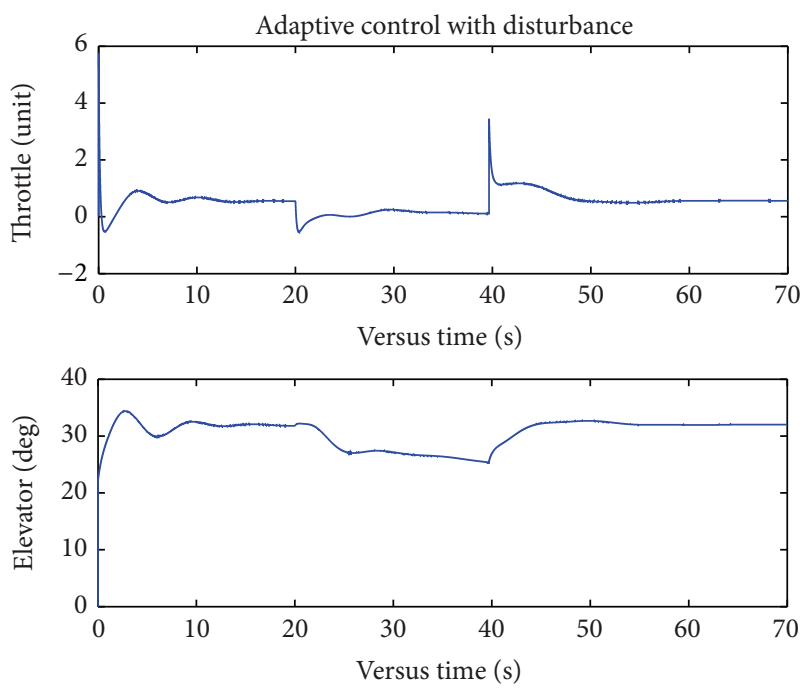

FIgURE 7: Final landing phase control signal.

\begin{tabular}{|c|c|}
\hline$I_{y}:$ & Moment of inertia in pitch \\
\hline$M:$ & The pitch moment \\
\hline$\delta_{e}, \delta_{t}:$ & $\begin{array}{l}\text { Elevator deflection bias angle and } \\
\text { engine throttle angle }\end{array}$ \\
\hline$M_{V_{0}}, D_{V_{0}}:$ & $\begin{array}{l}\text { Aerodynamic pitch moment and drag } \\
\text { derivative with respect to airspeed } V_{0}\end{array}$ \\
\hline$T_{V_{0}}, L_{V_{0}}:$ & $\begin{array}{l}\text { Thrust and aerodynamic lift derivative } \\
\text { with respect to airspeed } V_{0}\end{array}$ \\
\hline$M_{\delta_{e}}, L_{\delta_{e}}:$ & $\begin{array}{l}\text { Aerodynamic pitch moment and lift } \\
\text { derivative with respect to } \delta_{e}\end{array}$ \\
\hline$V_{w}, u_{x}, u_{y}:$ & $\begin{array}{l}\text { Turbulence velocity and body axis } \\
\text { components of } V_{w}\end{array}$ \\
\hline$V_{0}, \alpha_{0}, \dot{\alpha}_{0}, q_{0}, h_{0}:$ & The trim value of aircraft state \\
\hline$M_{V_{0}}, D_{V_{0}}:$ & $\begin{array}{l}\text { Aerodynamic pitch moment and drag } \\
\text { derivative with respect to airspeed } V_{0}\end{array}$ \\
\hline$T_{V_{0}}, L_{V_{0}}$ & $\begin{array}{l}\text { Thrust and aerodynamic lift derivatives } \\
\text { with respect to airspeed } V_{0}\end{array}$ \\
\hline$M_{\delta_{e}}, L_{\delta_{e}}:$ & $\begin{array}{l}\text { Aerodynamic pitch moment and lift } \\
\text { derivative with respect to elevator }\end{array}$ \\
\hline$D_{\alpha_{0}}, D_{h_{0}}:$ & $\begin{array}{l}\text { Aerodynamic drag derivative with } \\
\text { respect to } \alpha_{0} \text { and } h_{0}\end{array}$ \\
\hline$D_{\delta_{e}}:$ & Aerodynamic drag due to $\delta_{e}$ \\
\hline$M_{\alpha_{0}}, M_{\dot{\alpha}_{0}}:$ & $\begin{array}{l}\text { Aerodynamic pitch moment with } \\
\text { respect to } \alpha_{0} \text { and } \dot{\alpha}_{0}\end{array}$ \\
\hline$M_{q_{0}}:$ & $\begin{array}{l}\text { Aerodynamic pitch moment with } \\
\text { respect to } q_{0}\end{array}$ \\
\hline$T_{0}:$ & $\begin{array}{l}\text { Benchmark aerodynamic thrust at the } \\
\text { airspeed } V_{0}\end{array}$ \\
\hline$T_{\delta_{t}}:$ & $\begin{array}{l}\text { Aerodynamic thrust derivatives with } \\
\text { respect to the throttle }\end{array}$ \\
\hline$L_{\alpha_{0}}, L_{h_{0}}:$ & $\begin{array}{l}\text { Aerodynamic lift derivative with respect } \\
\text { to angle of attack and height. }\end{array}$ \\
\hline
\end{tabular}

\section{Competing Interests}

The authors declare that they have no competing interests.

\section{References}

[1] J. M. Urnes, R. K. Hess, R. F. Moomaw, and R. W. Huff, "Hdot automatic carrier landing system for approach control in turbulence," Journal of Guidance, Control, and Dynamics, vol. 4, no. 2, pp. 177-183, 1981.

[2] A. L. Prickett and C. J. Parkes, "Flight testing of the F/A-18E/F automatic carrier landing system," in Proceedings of the IEEE Aerospace Conference, pp. 52593-52612, March 2001.

[3] S. Yuan and Y. Yang, "Design of automatic carrier landing system using $H_{\infty}$ control," in Proceedings of the 3rd World Congress on Intelligent Control and Automation, pp. 3449-3451, IEEE, Hefei, China, June 2000.

[4] R. J. Niewoehner and I. I. Kaminer, "Design of an autoland controller for an F-14 aircraft using $H_{\infty}$ synthesis," Journal of Guidance, Control, and Dynamics, vol. 19, no. 3, pp. 656-663, 1996.

[5] M. Steinberg, "Development and simulation of An F/A-18. Fuzzy logic automatic carrier landing system," in Proceedings of the 2nd IEEE International Conference on Fuzzy Systems, pp. 797-802, April 1993.

[6] I. Fialho, G. I. Balas, and J. Renfrow, "On the design of LPV controllers for the F-14 aircraft lateral-directional axis during powered approach," in Proceedings of the American Control Conference, pp. 123-127, June 1997.

[7] N. A. Denison, Automated Carrier Landing of an Unmanned Combat Aerial Vehicle Using Dynamic Inversion, Department of Aeronautical and Astronautical Engineering Graduate School of Engineering and Management, Air Force Institute of Technology, Air Force University, 2007.

[8] G. Tao, Adaptive Control Design and Analysis, John Wiley \& Sons, Hoboken, NJ, USA, 2003.

[9] E. Lavretsky, "Combined/composite model reference adaptive conrol," in Proceedings of the AIAA Guidance, Navigation, and Control Conference, AIAA 2009-6065, Chicago, Ill, USA, August 2009.

[10] H. I. Basturk and M. Krstic, "State derivative feedback for adaptive cancellation of unmatched disturbances in unknown strict-feedback LTI systems," Automatica, vol. 50, no. 10, pp. 2539-2545, 2014.

[11] H. I. Basturk and M. Krstic, "Adaptive sinusoidal disturbance cancellation for unknown LTI systems despite input delay," Automatica, vol. 58, pp. 131-138, 2015.

[12] X. Y. Guo and M. Bodson, "Equivalence between adaptive feedforward cancellation and disturbance rejection using the internal model principle," International Journal of Adaptive Control and Signal Processing, vol. 24, no. 3, pp. 211-218, 2010.

[13] X. Chen, "Adaptive sliding mode control for discrete-time multi-input multi-output systems," Automatica, vol. 42, no. 3, pp. 427-435, 2006.

[14] Z. Zhao, J. Zhang, L. Sun, and D. Zhang, "Terminal sliding mode control with adaptive law for uncertain nonlinear system," Mathematical Problems in Engineering, vol. 2015, Article ID 519090, 7 pages, 2015.

[15] Y. Guo, S.-M. Song, and X.-H. Li, “Terminal sliding mode control for attitude tracking of spacecraft based on rotation matrix," Mathematical Problems in Engineering, vol. 2015, Article ID 187924, 9 pages, 2015.

[16] Q. Zou, F. Wang, and Q. Zong, "Nonlinear constrained adaptive backstepping tracking control for a hypersonic vehicle with uncertainty," Mathematical Problems in Engineering, vol. 2015, Article ID 237453, 16 pages, 2015. 
[17] X.-X. Yin, Y.-G. Lin, W. Li, H.-W. Liu, and Y.-J. Gu, "Adaptive sliding mode back-stepping pitch angle control of a variabledisplacement pump controlled pitch system for wind turbines," ISA Transactions, vol. 58, pp. 629-634, 2015.

[18] N. T. Nguyen, "Optimal control modification for robust adaptive control with large adaptive gain," Systems \& Control Letters, vol. 61, no. 4, pp. 485-494, 2012.

[19] J.-Z. Geng, H.-L. Yao, and H. Zhang, "Studies on effect of air wake on slope and landing property of carrier aircraft," Journal of System Simulation, vol. 21, no. 18, pp. 5940-5943, 2009.

[20] D. D. Boskovic and J. Redding, "An autonomous carrier landing system for unmanned aerial vehicles," in Proceedings of the AIAA Conference on Guidance, Navigation, and Control, AIAA 2009-6264, Chicago, Ill, USA, August 2009.

[21] "Department of defense handbook flying qualities of piloted aircraft. MIL-STD-1797A," Department of Defense, USA, 1995.

[22] B. Etkin, Dynamics of Atmospheric Flight, John Wiley \& Sons, New York, NY, USA, 1972.

[23] M. V. Cook, Flight Dynamics Principles, Butterworth-Heinemann, London, UK, 2007.

[24] A. K. Imai, R. R. Costa, L. Hsu, G. Tao, and P. V. Kokotovic, "Multivariable adaptive control using high-frequency gain matrix factorization," IEEE Transactions on Automatic Control, vol. 49, no. 7, pp. 1152-1157, 2004.

[25] L. Wen, G. Tao, and H. Yang, "Adaptive turbulence compensation for multivariable nonlinear aircraft models," in Proceedings of the American Control Conference (ACC '15), pp. 5557-5562, July 2015.

[26] B. L. Stevens and F. L. Lewis, Aircraft Control and Simulation, Wiley-Interscience, New York, NY, USA, 2003. 


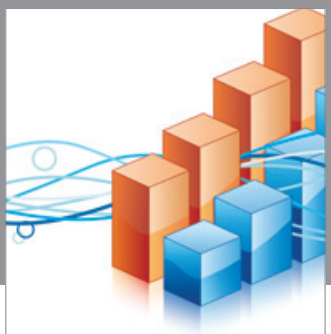

Advances in

Operations Research

vatem alat4

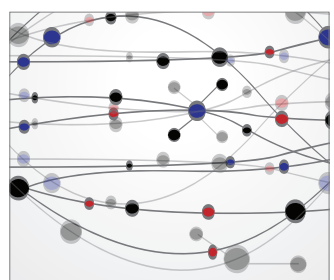

\section{The Scientific} World Journal
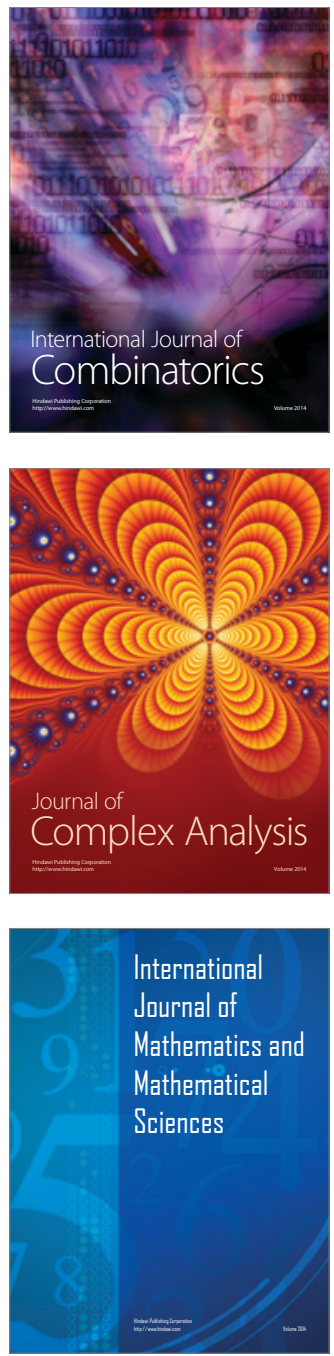
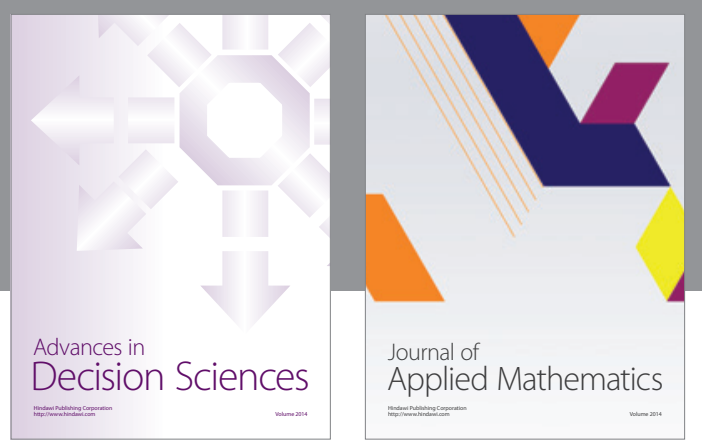

Algebra

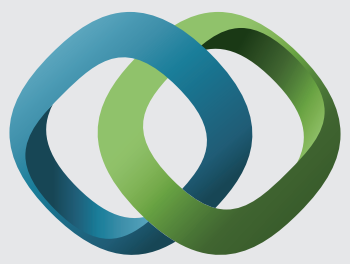

\section{Hindawi}

Submit your manuscripts at

http://www.hindawi.com
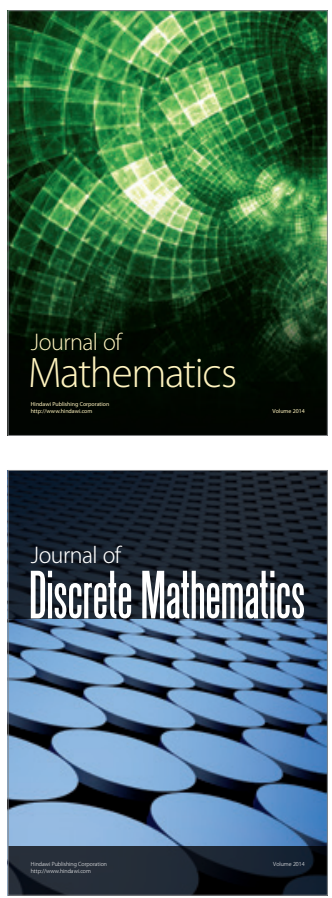

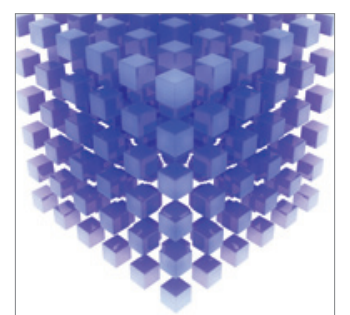

Mathematical Problems in Engineering
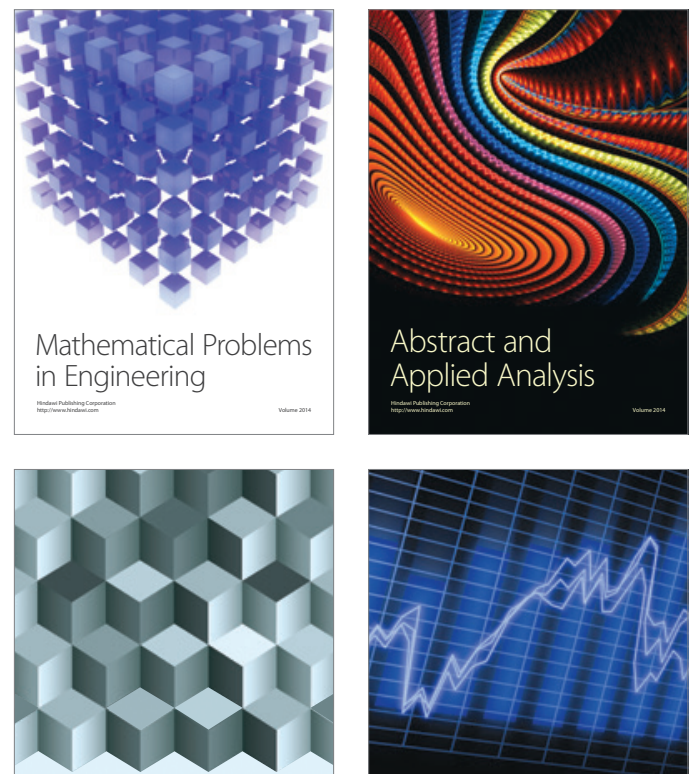

Journal of

Function Spaces

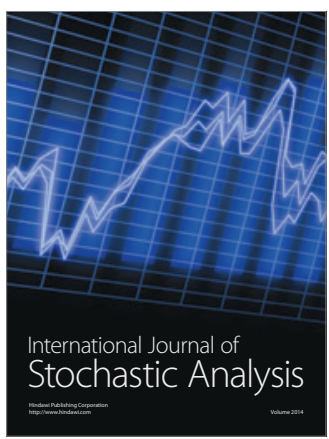

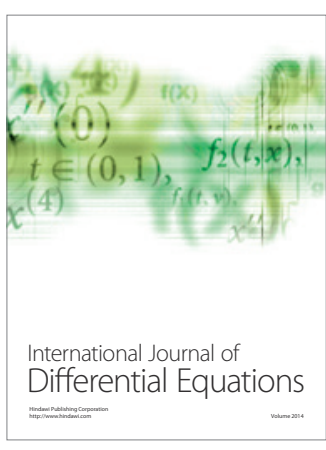
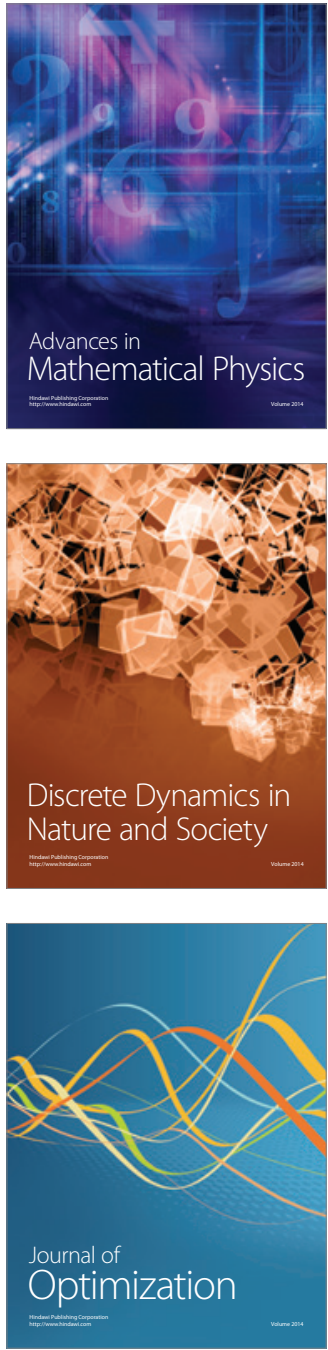